\title{
FIB, TEM and AFM Quantitative Investigation of Nanostructure and Nanoscale Friction Properties of Single Partially Fluorinated Carbon Nanofibres
}

\author{
Philippe Bilas ${ }^{1}$, Jean-Louis Mansot ${ }^{1,2}$, Laurent Legras ${ }^{3}$, Philippe Thomas ${ }^{1}$, Marc Dubois ${ }^{4}$, André \\ Hamwi $^{4}$ and Katia Guerin ${ }^{4}$ \\ 1. Groupe de Technologie des Surfaces et des Interfaces (GTSI EA 2432) \\ 2. Centre Commun de Caractérisation des Matériaux des Antilles et de la Guyane, Université des Antilles \\ et de la Guyane, Campus Fouillole, BP 25097157 Pointe à Pitre - Guadeloupe-France- French West \\ Indies \\ 3. EDF - R\&D, Department MMC and MAI, Avenue des Renardières, Les Renardiéres, 77818 Moret sur \\ Loing Cedex \\ ${ }^{4 .}$ Clermont Université, Université Blaise Pascal, Institut de Chimie de Clermont-Ferrand (ICCF, UMR \\ 6296), Inorganic Materials team, 63177 Aubière, France
}

New lubrication strategies in order to reduce friction and wear in engines consist in the addition, in lubricants, of lubricating solid nanoparticles [1]. Among the great variety of available nanoparticles, graphitized carbon nanofibres revealed interesting tribologic properties which are strongly improved when they are partially fluorinated [2]. The present work is concerned with the analytical study of the structure and composition of partially fluorinated carbon nanofibres and with the investigation of their friction properties at nanoscale in order to better understand their macroscopic tribologic behaviour.

High purity $(>90 \%$ ) carbon nanofibres of $0.2 \mu \mathrm{m}$ diameter and few $\mu \mathrm{m}$ length, supplied by MER Corporation are first graphitized by heating at $1800^{\circ} \mathrm{C}$ in argon atmosphere. Their fluorination is then carried out under pure fluorine gas flow in a Monel reactor at $420^{\circ} \mathrm{C}$ during $16 \mathrm{~h}$. The atomic $\mathrm{F} / \mathrm{C}$ ratio (0.15) is determined by ${ }^{19} \mathrm{~F}$ solid NMR [2]. The Fluorinated Carbon Nano Fibres will be noted FCNFs. A small amount of FCNFs ( $2 \mathrm{mg}$ ) is embedded into epoxy resin. For TEM characterization, ultrathin sections (60 nm thick) of the epoxy/fibres block are prepared using ultramicrotomy (Leica Ultracut). The remaining block is then introduced in a dual beam (FEI Helios Nanolab) in order to cut the fibers emerging from the epoxy. The amorphous layer introduced by FIB etching is removed in a plasma cleaner using oxygen atmosphere. The as prepared block is then directly used for SEM and AFM studies.

SEM studies are carried out in low vacuum $(130 \mathrm{~Pa})$ conditions on a FEI Quanta 250 ESEM equipped with a tungsten cathode running at $5 \mathrm{kV}$. TEM investigations of the structure and composition of the FCNFs are carried out using a FEI Tecnaï F 20 X twin running at $200 \mathrm{kV}$ fitted with an EDAX SiLi detector and a post column Gatan Quantum energy filter.

Topography (AFM) and friction behaviour at nanoscale (LFM) are investigated with a silicon tip under air, at $25^{\circ} \mathrm{C}-55 \%$ relative humidity, in constant force mode using a PICO scanning probe microscope from Molecular Imaging Corp. The quantitative measurements of the forces are done using the method developed in previous works [3].

Typical images and EDS analyses carried out onto FCNFs cross sections (figure 1) clearly reveal the graphitized structure [(002) $3.4 \AA$ graphite lattice] of the core of the fibres whereas the outer shell presents $4.5 \AA$ inter reticular distances corresponding to $(\mathrm{CF})$ n fluorinated phase. The fluorine profile recorded along a diameter of the section clearly points out that fluorination only interest a $30-50 \mathrm{~nm}$ thick outershell. The AFM topographic image show a differential etching of the nanofibre resulting 
probably from the higher etching rate of the fluorinated part. The friction image acquired across the fiber and corresponding friction profile quantitatively deduced, taking into account the outershell slope, clearly reveal that friction coefficient of the external fluorinated part ( $\mu=$ ??????) is higher than the pristine graphitic core $(\mu=0.20 \pm 0.04)$. This results are consistent with the friction nanoproperties of standard HOPG $(\mu=0.007 \pm 0.003)$ and perfluorinated HOPG $(\mu=0.65 \pm 0.05)$ also measured in this work.

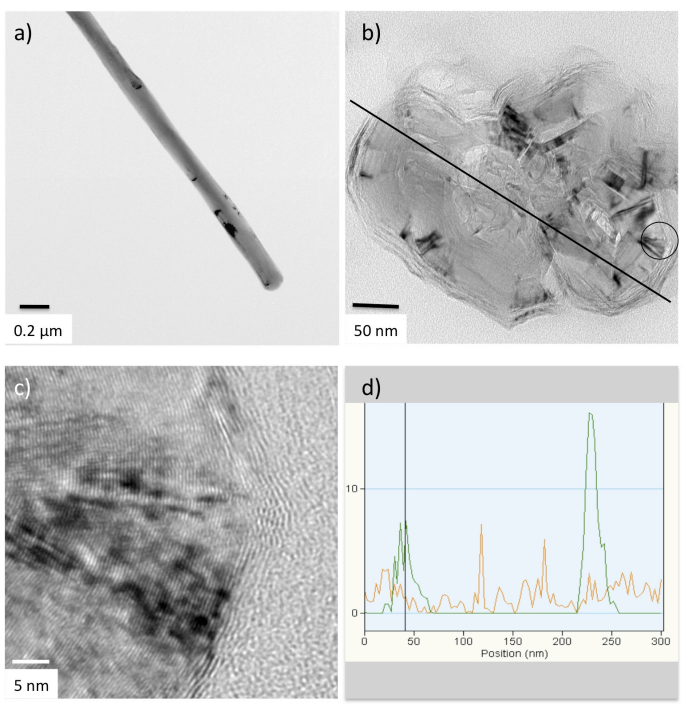

Figure 1. ATEM fiber structure and composition investigations of a nanofiber. a) morphology of the fiber. b-c) Cross section showing $3.4 \AA$ carbon lattice and $4.5 \AA$ fluorinated carbon lattice in the outershell. d) oxygen and fluorine EDS profiles acquire along a diameter (line in $b$ ) of the cross section pointing out the fluorine presence only on a $50 \mathrm{~nm}$ outer shell.

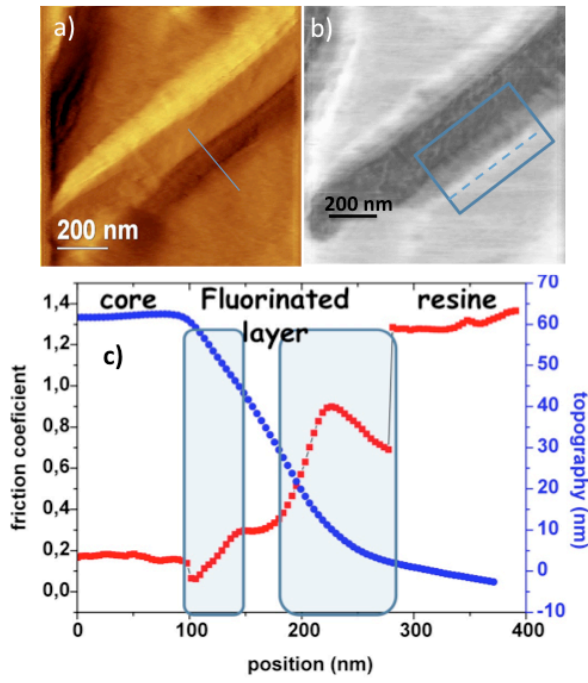

Figure 2. a-b) AFM topographic and friction images collected on a single fluorinated carbon nanofiber partially eched by FIB and plasma cleaning treatment. c) topographic profile across the fiber corresponding to the superimposed line (a) and friction coefficient profile collected on the area underlined by the rectangle (b).

The nanofriction behaviour of the different part of the FCNFs do not agree with their macrotribologic properties as far as friction of nanofibres is improved by fluorination. This can be explained by the fact that at macroscopic level friction process is probably governed by inter fiber interactions whereas in this study it is governed by the $\mathrm{Si} / \mathrm{FCNF}$ interactions. Nano experiments are under development to properly reproduce the single FCNF/FCNF interactions.

Authors acknowledge the Ministère de la Recherche, the Conseil Régional de la Guadeloupe, the Conseil Régional de la Martinique, the Fonds Social Européen (FSE) and Fonds Européens de Développement Régional (FEDER) for their financial supports.

\section{References:}

[1] J.L Mansot, J.M Martin, Y Bercion, L Romana, "Nanolubrication”, Brazil. J. of Phys. vol. 39 n¹ (2009), p.186.

[2] P Thomas, K Delbe, D Himmel, J.L Mansot, M Dubois, K Guerin, W Zhang, A Hamwi, "Tribological properties of fluorinated carbon nanofibres", Tribology Letters vol 33 n³ (2009) p.49. [3] P Bilas, L Romana, B Kraus, Y Bercion, J.L Mansot, "Quantitative characterization of friction coefficient using lateral force microscope in the wearless regime", Review of Scientific Instrument Vol $75 \mathrm{~N}^{\circ} 2$ (2004) p.415. 